\title{
Dual dopants In2S3/ZnS quantum dots for targeted fluorescence and magnetic resonance imaging
}

\author{
Jia-Yaw Chang, Pei-Yu Lai
}

Department of Chemical Engineering, National Taiwan University of Science and Technology, Taiwan

In this work, we describe a rapid microwave-assisted synthesis of $\mathrm{Ag}+$ and $\mathrm{Mn} 2+$ dual-dopants $\mathrm{In} 2 \mathrm{~S} 3 / \mathrm{ZnS}$ quantum dots (Qdots), which integrate functions of optical fluorescence and magnetic resonance imaging. Ag+ dopants were used to alter the optical properties of the In2S3 host, whereas $\mathrm{Mn} 2+$ co-dopants with their unpaired electrons provided paramagnetic properties. The emission wavelength of the core/shell Qdots could be tuned from 550 to $743 \mathrm{~nm}$ with a quantum yield of $45 \%$. In vitro targeted cell luminescence and magnetic resonance imaging showed that Qdots conjugated to hyaluronic acid was selectively and efficiently internalized in CD44-expressing tumor cells, confirming that the resultant Qdots could function as dual-modal imaging probes for accurate diagnosis. Additionally, the obtained Qdots exhibited negligible cytotoxicity in vitro or in vivo, which rendered them promising diagnostic probes for dual-modal imaging in clinical applications. 